\title{
QPACE 2 and Domain Decomposition on the Intel Xeon Phi*
}

\author{
Paul Arts $^{a}$, Jacques Bloch ${ }^{b}$, Peter Georg ${ }^{b}$, Benjamin Glässle ${ }^{b}$, Simon Heybrock ${ }^{b}$,

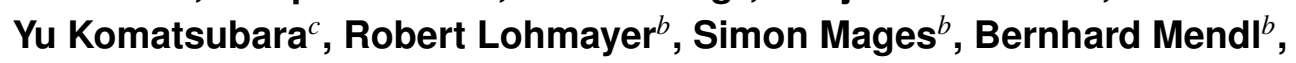 \\ Nils Meyer ${ }^{b}$, Alessio Parcianello ${ }^{a}$, Dirk Pleiter ${ }^{b, d}$, Florian Rappl $^{b}$, Mauro Rossi ${ }^{a}$, \\ Stefan Solbrig ${ }^{b}$, Giampietro Tecchiolli ${ }^{a}$, Tilo Wettig ${ }^{\dagger b}$, Gianpaolo Zanier ${ }^{a}$ \\ ${ }^{a}$ Eurotech HPC, Via F. Solari 3/A, 33020 Amaro, Italy \\ ${ }^{b}$ Department of Physics, University of Regensburg, 93040 Regensburg, Germany \\ ${ }^{c}$ Advanet Inc., 616-4 Tanaka, Kita-ku, Okayama 700-0951, Japan \\ ${ }^{d}$ JSC, Jülich Research Centre, 52425 Jülich, Germany \\ E-mail: tilo.wettig@ur.de
}

We give an overview of QPACE 2, which is a custom-designed supercomputer based on Intel Xeon Phi processors, developed in a collaboration of Regensburg University and Eurotech. We give some general recommendations for how to write high-performance code for the Xeon Phi and then discuss our implementation of a domain-decomposition-based solver and present a number of benchmarks.

The 32nd International Symposium on Lattice Field Theory,

23-28 June, 2014

Columbia University, New York, NY

${ }^{*}$ Work supported by DFG in the framework of SFB/TRR-55.

† Speaker. 


\section{Introduction}

After the invention of lattice QCD in 1974 [1] it quickly became clear that (i) it had great potential to yield physically relevant nonperturbative results and (ii) enormous computational resources would be required to obtain them. Such resources were not readily available at the time. This led some of the physicists involved to design and build special supercomputers optimized for lattice QCD simulations. These activities started in the 1980s and are continuing until now. The resulting machines have been serving as work horses for large-scale lattice QCD simulations worldwide and were typically much more cost-efficient than commercially available machines. They also influenced the design of commercial supercomputers such as BlueGene. A probably incomplete list of such machines includes ACPMAPS, GF11, Fermi-256, QCDSP, QCDOC (all in the US), QCDPAX, CP-PACS, PACS-CS (Japan), as well as APE100, APEmille, apeNEXT, and QPACE 1 (Europe), see also [2] for a review.

However, in the past several years the situation has changed. First, the development of specialized processor chips has become too expensive for academic projects. Second, commercial supercomputers such as BlueGene scale well for lattice QCD and are reasonably cost- and energyeffective (although the development of truly scalable machines such as BlueGene or the K computer relies on enormous government funding that is not always forthcoming). Finally, standard compute clusters (typically with accelerators) are now readily available and can be used for capacity-type problems where strong scalability is not a must.

Nevertheless, it still makes sense to pursue academic hardware-development activities by combining commercially available components in an innovative way, in particular regarding the communication between processors. This can result in machines that are more scalable and more costand energy-effective than commercial machines. The development activities typically involve industry partners that also benefit from the innovative concepts originating on the academic side ("codesign"). In our most recent projects these partners were IBM (QPACE 1 [3]) and Intel (QPACE 2) as well as Eurotech (QPACE 1 and 2). Note that the current "custom" designs for lattice QCD are suitable for a broader application portfolio than they used to be in the past. Note also that hardware development is only part of the story and that system software and high-performance application codes are equally essential (and in fact absorb a large fraction of the development activities).

In Sec. 2 we give an overview of the QPACE 2 project, which is based on the Knights Corner (KNC) version of the Intel Xeon Phi processor. We also discuss in some detail our experiences with this new "many-core" architecture and give recommendations for programming it. In Sec. 3 we present a new solver code tailored to the KNC. This code is based on domain decomposition (DD), which is less bandwidth bound and more latency tolerant than other approaches, and thus leads to better scaling behavior [4]. In Sec. 4 we summarize and give an outlook to QPACE 3, which will be an upgrade of the QPACE 2 prototype system, based on the future Knights Landing (KNL) version of the Xeon Phi.

\section{Overview of QPACE 2}

Between QPACE 1 and QPACE 2, the acronym QPACE changed its meaning from "QCD Parallel Computing on the Cell Processor" to "QCD Parallel Computing Engine". The QPACE 2 

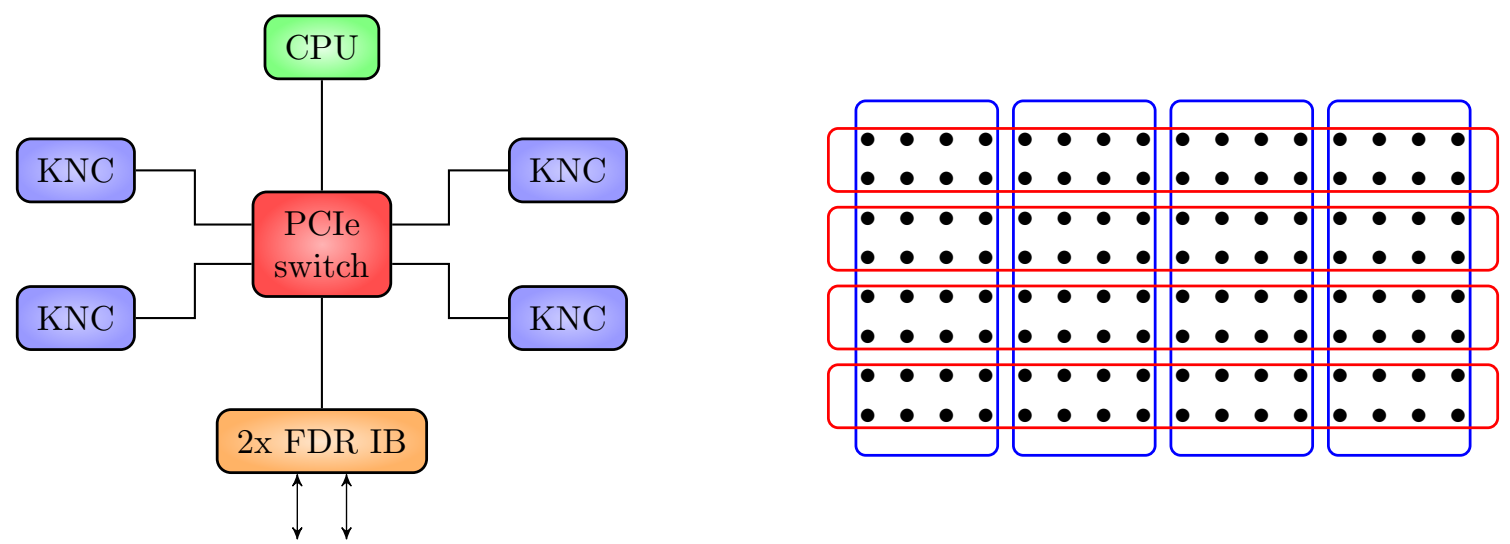

Figure 1: Left: QPACE 2 node design, see text for details. Right: Sketch of the two-dimensional hypercrossbar network (based on FDR Infiniband) of QPACE 2, here shown for $16 \times 8$ nodes as an example. Each dot represents a node with two IB ports that is connected to one IB switch each in the $x$-and $y$-directions. The switches, which here have 32 ports each, are indicated by the red and blue squares, respectively.

project is funded by the German Research Foundation (DFG) and led by the University of Regensburg in collaboration with Eurotech (Italy/Japan) and Intel, with additional contributions from the Jülich Supercomputing Center and the University of Wuppertal. ${ }^{1}$

\subsection{Node design}

Like most supercomputers, QPACE 2 consists of many identical nodes connected by a network. The node design is shown in Fig. 1 (left). Four Intel Xeon Phi 7120X processors (a.k.a. Knights Corner or KNC) are connected to a PCIe switch (PEX 8796 by PLX). Also connected to the switch are a low-power CPU (Intel Xeon E3-1230L v3) and a dual-port FDR Infiniband card (Mellanox Connect-IB). The CPU serves as the PCIe root complex. The KNCs as well as the IB card are PCIe endpoints. Peer-to-peer (P2P) communication between any pair of endpoints can take place via the switch. The rationale behind this node design is that a high-performance network is typically quite expensive. A "fat" node with several processing elements and cheap internal communications (here over PCIe) has a smaller surface-to-volume ratio and thus requires less network bandwidth per floating-point performance, which lowers the relative cost of the network. The number of KNCs and IB cards on the PCIe switch is determined by the number of lanes supported by commercially available switches and by the communication requirements within and outside of the node. We are using the largest available switch, which supports 96 lanes PCIe Gen3. Each of the KNCs has a 16-lane Gen2 interface (corresponding to a bandwidth of $8 \mathrm{~GB} / \mathrm{s}$ ), while both the CPU and the IB card have a 16-lane Gen3 interface (i.e., almost $16 \mathrm{~GB} / \mathrm{s}$ each). The external IB bandwidth for two FDR ports is $13.6 \mathrm{~GB} / \mathrm{s}$. This balance of internal and external bandwidth is consistent with the communication requirements of Lattice QCD, see also Sec. 3. Each of the KNCs has 61 cores, a clock speed of $1.238 \mathrm{GHz}$, a peak performance of $1.2 \mathrm{TFlop} / \mathrm{s}$ (double pre-

\footnotetext{
${ }^{1}$ The development of QPACE 2 was initially pursued in collaboration with T-Platforms. Unfortunately, this collaboration was terminated after T-Platforms was placed on the so-called Entity List of the US Department of Commerce in March 2013 and no longer had access to US technology, such as Intel Xeon Phi processors. This caused a significant delay for QPACE 2. Note that T-Platforms was removed from the Entity List in December 2013.
} 
cision), and 16 GB of GDDR5 memory with a peak bandwidth of $352 \mathrm{~GB} / \mathrm{s}$. All of these numbers are nominal. Sustained numbers will be discussed in Sec. 2.5.

One advantage of our node design is that the KNCs could in principle be replaced by GPUs, although in this case a stronger CPU would be advisable.

\subsection{Network}

The nodes are connected by an IB-based network with a hyper-crossbar topology. This topology was introduced by the CP-PACS collaboration [5]. For QPACE 2 we use a two-dimensional version as indicated in Fig. 1 (right). In general, if we have nodes with $d$ ports each and switches with $p$ ports each, a $d$-dimensional hyper-crossbar has a maximum size of $p^{d}$ nodes. We are using IB edge switches with 36 ports, $p=32$ of which are used for connecting the nodes. Thus our maximum partition size is 1024 nodes, corresponding to $4096 \mathrm{KNCs}$ (which is sufficient for present-day lattice sizes and more than our budget can afford). The remaining 4 switch ports can be used for connecting the machine to a storage system and/or for connecting the switches in a torus (using the torus-2QoS routing engine in the OpenSM subnet manager).

The advantage of a hyper-crossbar over a torus is that we have full connectivity in every single dimension with one switch hop, and all-to-all connectivity by going through at most one intermediate node. The advantage over a (fat) tree is lower cost. We note for completeness that a higher-dimensional application can be mapped to a lower-dimensional hyper-crossbar in a variety of ways. Typically this should be done such that the communication requirements are minimized.

\subsection{System design}

The components of a node are integrated in what we currently call a "brick". ${ }^{2}$ The basis of a brick is a midplane, see Fig. 2 (left). This midplane accommodates the PCIe switch, a power connector and a number of power converters, and six slots for standard PCIe form factor cards, i.e., a CPU card (Fig. 2 middle), a Connect-IB card (Fig. 2 right), and four KNC cards (Fig. 3). The KNC card (by Intel) and the Connect-IB card (by Mellanox) are commercial components. The midplane and the CPU card were designed for QPACE 2 but can be reused for other projects/products. The CPU card also contains 16 GB of DDR3 memory, a platform controller hub (PCH), and a baseboard management controller (BMC). It provides two Ethernet and several other interfaces such as USB, VGA, and SPI. One of the Ethernet interfaces is used by the BMC and the other by the CPU to form two separate Ethernet networks. The BMC network is used for low-level control of the nodes. The CPU network is used for booting the operating system and for login. Both networks are also used for system monitoring.

The cooling concept of QPACE 2 is a novel idea based on roll-bond technology (see p. 253 and Fig. 8.23 of [6] for an explanation of this technology). The concept is shown in Fig. 3 for a KNC card. The other cards as well as the midplane are also cooled in this way. Water flows through a roll-bond plate (made of aluminum) which is thermally coupled to the hot components via interposers (made of aluminum or copper) and thermal grease or thermal interface material.

\footnotetext{
${ }^{2}$ This name is due to the obvious similarity of our mechanical design, see Fig. 5, with a brick used in the construction industry, but we should probably change it in the future to avoid confusion with the alternative meaning of "non-functioning electronic device".
} 

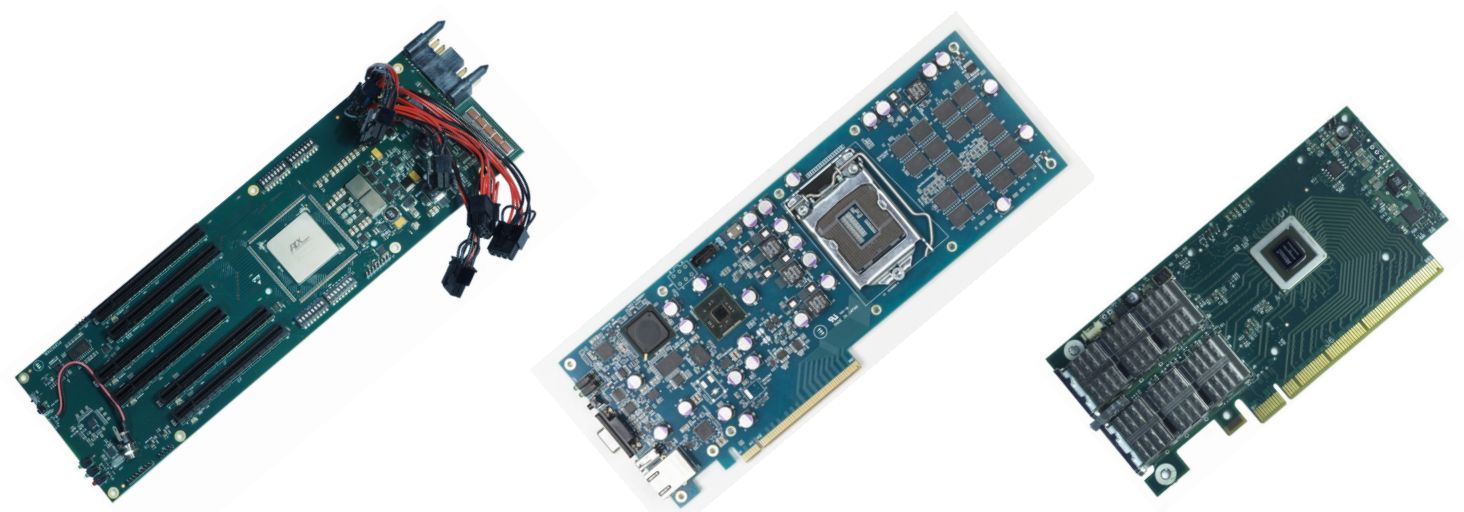

Figure 2: Some components of a QPACE 2 node (not to scale): Midplane (left), CPU card (middle), and Connect-IB card (right). The power cables on the midplane are needed for the KNCs. See text for details.
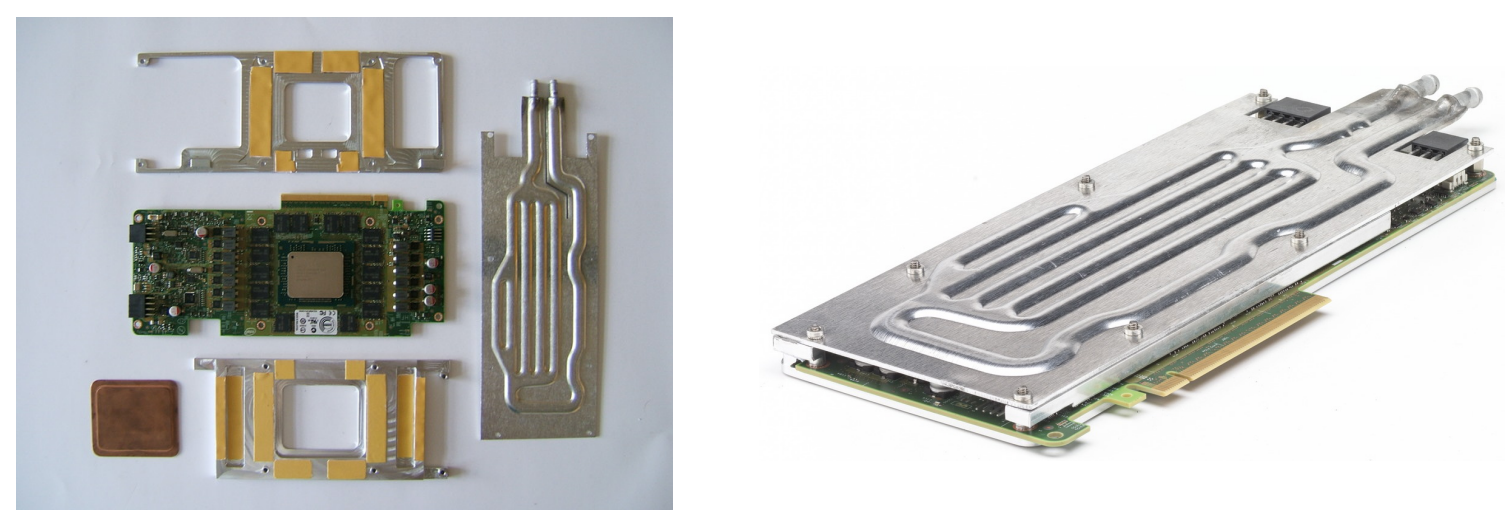

Figure 3: QPACE 2 cooling concept, here for the KNC card. Left: The hot components of the card (middle) are thermally coupled to the roll-bond plate (right) via interposers (top and bottom). The copper plate is inserted in the hole of the interposer and interfaces with the KNC chip through thermal grease. The yellow pieces are thermal interface material. Right: Fully assembled water-cooled KNC card. The top interposer is coupled to the roll-bond plate with thermally conductive glue. The bottom interposer, which cools memory chips on the bottom of the card, is thermally coupled to the top interposer via aluminum surfaces (visible in Fig. 4 right) and screws.

The advantage of the roll-bond technology is that it is cheap and widely available and that customshaped water channels are very easy to implement. In addition, the risk of leakages is minimized. With other technologies it is much harder or more expensive to create channels of arbitrary shape and to make sure that there are no leakages. Our design allows for cooling water temperatures of at least $50^{\circ} \mathrm{C}$, which makes free cooling possible year-round. Thus no chillers are needed, which in our installation improves the overall energy efficiency of the machine significantly.

Once the cooling solutions have been assembled, the six PCIe cards are plugged into the midplane, as shown in Fig. 4. The six roll-bond plates are connected to a mini-manifold with plastic tubes. The entire assembly is then put into a housing, see Fig. 5. The mini-manifold is visible in Fig. 5 (right). It is connected to the cooling-water circuit via drip-free quick-disconnect couplings. The lever visible in Fig. 5 (left) locks the power connector and the quick disconnects into place in the rack. 

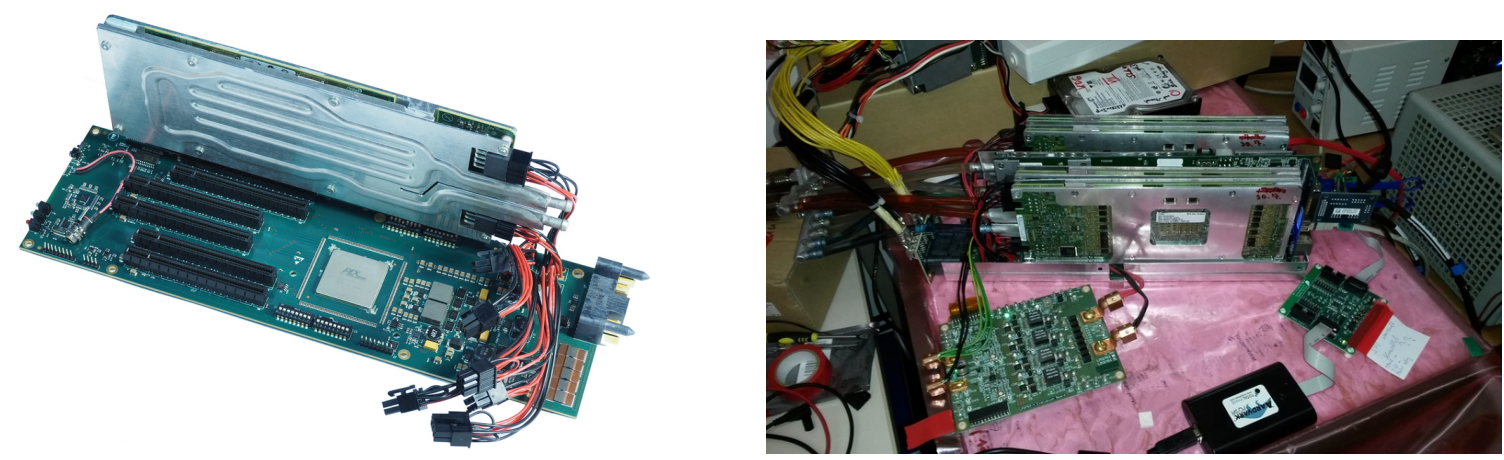

Figure 4: Left: The PCIe cards with their cooling assembly are plugged into the midplane. Here only one KNC card is shown. Right: One node (with all six cards liquid-cooled but without housing) is undergoing testing.
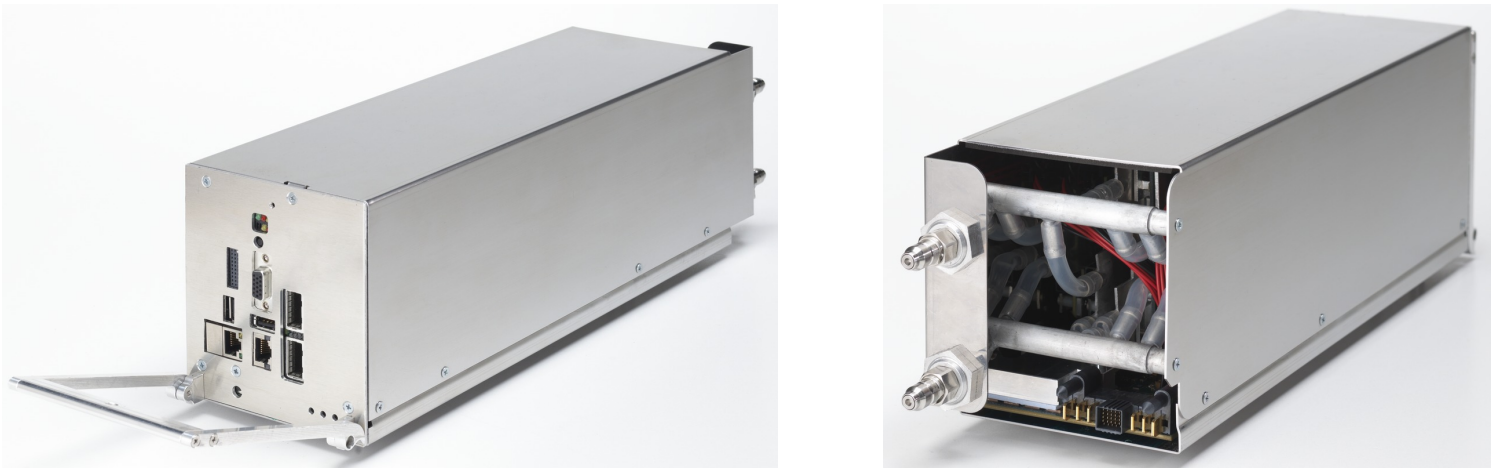

Figure 5: Left: A fully assembled node in its housing. Several interfaces are provided on the front panel: dual FDR Infiniband, dual Ethernet, USB, VGA, and debug connector. Right: Back side of the brick with power connector, mini-manifold, and drip-free quick-disconnect couplings (male parts) for in- and outlet.

We use a standard 19-inch rack of height $42 \mathrm{U}$. The height of a brick is $3 \mathrm{U}$, and we can put $4+4$ bricks in $3 \mathrm{U}$ ( 4 from the front of the rack, 4 from the back). This translates to 64 nodes, i.e., $256 \mathrm{KNCs}$, in $24 \mathrm{U}$. The remaining space in the rack is used for the cooling-water distribution, power distribution, 3 Ethernet and 4 Infiniband switches, a management/login server, and cable management. The peak performance of a rack is about $310 \mathrm{TFlop} / \mathrm{s}$ (double precision).

$12 \mathrm{~V}$ power is delivered to the nodes via massive power bars made of copper and small power backplanes, see Fig. 6. The latter also contain a DIP switch that is used to assign a unique location ID to each brick. Based on this location ID we then define IP addresses. The power bars are fed by industry-standard platinum power supply units (PSUs) that convert $230 \mathrm{~V}$ AC to $12 \mathrm{~V} \mathrm{DC}$ at about 95\% efficiency, see Fig. 7 (left). Each PSU can deliver up to $2 \mathrm{~kW}$. We subdivide the rack power distribution into 8 domains, with each domain powered by 6 PSUs serving 8 bricks in parallel. Assuming a typical power consumption of $1 \mathrm{~kW}$ per node, we are just on the border between $5+1$ and $4+2$ redundancy. A PSU control board designed in Regensburg monitors and controls the PSUs via PMBus, using a BeagleBone Black single-board computer that plugs into the master control board, see Fig. 7 (right). One PSU control board services 16 PSUs, hence we have 3 control boards for 48 PSUs. The PSUs can deliver a maximum power of $96 \mathrm{~kW}$ per rack. First 

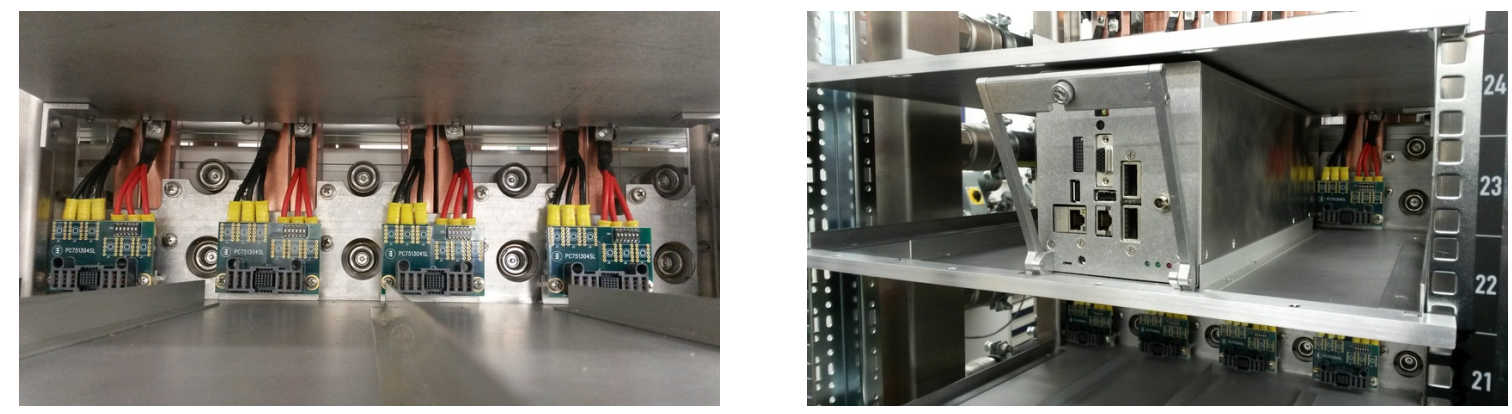

Figure 6: Left: Power bars with power backplanes attached via cables. Also visible are the quick-disconnect couplings (female parts) for in- and outlet. Right: A brick (of height $3 \mathrm{U}$ ) is being inserted into its slot.
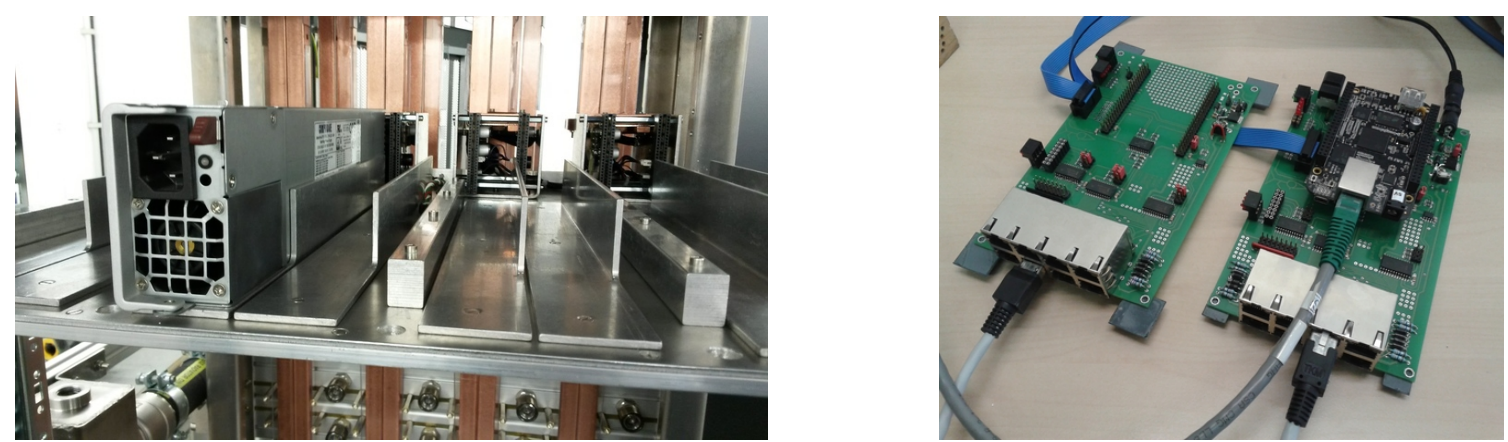

Figure 7: Left: The PSUs are connected to the power bars via power distribution boards (PDB). Each PDB holds two PSUs. Here, only a single PSU is shown. Right: The master PSU control board (right) accommodates a BeagleBone Black single-board computer. The other PSU control boards (e.g., the one on the left) run in slave mode.

results suggest that synthetic benchmarks will consume up to $85 \mathrm{~kW}$, whereas high-performance QCD code will run at about $60 \mathrm{~kW}$.

\subsection{System software}

The BMC on the CPU card is running an embedded Linux version and boots from flash memory as soon as power is turned on. It supports the typical functionalities of a board management controller, e.g., it can hold the other devices in the brick in reset or release them, it can monitor voltages as well as current and temperature sensors and act accordingly (e.g., by performing an emergency shutdown), or it can access the registers in the PCIe switch via an $\mathrm{I}^{2} \mathrm{C}$ bus. Note that our nodes are diskless. Once the CPU is released from reset it PXE-boots a minimal Linux image over the Ethernet network. The full Linux operating system (currently CentOS 7.0) uses an NFSmounted root file system. The KNCs are booted and controlled by the CPU using Intel's KNC software stack MPSS. The KNCs support the Lustre file system of our main storage system, which is accessed over Infiniband. A variety of system monitoring tools are running either on the BMC (as described above) or on the CPU, which, for example, regularly checks the temperatures of all major devices (KNCs, CPU, Infiniband HCA, PCIe switch) as well as various error counters (ECC, PCIe, Infiniband). A front-end server is used to NFS-export the operating system, to communicate with the nodes and monitor them, to log in to the machine, and to control the batch queues. 


\subsection{KNC architecture and its implications for the programmer}

Let us first describe some relevant details of the KNC architecture, focusing on the $7120 \mathrm{X}$ version used in QPACE 2. The processor chip contains 61 compute cores running at $1.238 \mathrm{GHz}$. The cores are connected by a bidirectional ring bus, which also interfaces to the GDDR5 memory controllers (for a total of $16 \mathrm{~GB}$ of on-board memory) and the PCIe I/O logic (16 lanes PCIe Gen2). Each core has a 512-bit wide SIMD unit on which it can perform fused-multiply-add instructions in either single or double precision. This translates to a peak performance of $1.2 \mathrm{TFlop} / \mathrm{s}$ per KNC in double precision. There are four threads per core that have their own register set but shared execution units and caches. Each core has a private L2 cache of $512 \mathrm{kB}$. The L2 caches are kept coherent with a distributed tag directory, i.e., if core A needs data that are not in its L2 but in the L2 of core B, these data are automatically fetched from core B via the ring bus, rather than from memory. This is faster than memory access, but not by much in practice.

While the $\mathrm{KNC}$ architecture is quite promising, the actual hardware implementation has some performance-relevant pitfalls that one should be aware of. For example, instructions from a given thread can only be issued every other cycle. Therefore we need at least two threads to issue instructions in each cycle. Also, there is no L1 hardware prefetcher. Furthermore, the cores execute instructions in order, which implies that cache misses always stall execution of a thread for $10 \sim 100$ cycles. To hide these stalls we also need to use multiple threads.

After initial theoretical performance analysis, we have performed microbenchmarks and experimented heavily while implementing and optimizing our software. We believe to have arrived at a solid understanding of how to write high-performance code for the KNC. The purpose of this subsection is to describe some of the insights we have gained, starting from a single core and going to the full multi-node system.

For some parts of the application, such as the stencil computation for the Wilson Dirac operator, the small size of the $\mathrm{L} 1$ cache $(32 \mathrm{kB})$ can become a limiting factor. There are two reasons for this. First, while the L1 size has been kept constant, the size of the vector registers has been doubled compared to AVX (which in turn is double that of SSE). As a consequence the size of the working set is typically larger, which leads to more L1 capacity cache misses, i.e., data are more frequently evicted from L1 before they can be reused. Second, to fully use a core we need to use two threads, and to hide stalls due to cache misses we would often like to use three or four. Thus the L1 size per thread is less than $32 \mathrm{kB}$. The 8-way associativity of the L1 may further reduce the available space per thread since multiple threads compete for the 8 ways (unless they work on the same data). The lack of an L1 hardware prefetcher also means that software prefetching can be crucial. With well-placed L1 software prefetches ${ }^{3}$ we often get good results with two threads. Without optimized L1 prefetching typically three or four threads yield the best performance, at the cost of incurring the negative effects described above.

The L2 cache is coherent but not shared between cores. This has several consequences. In a typical stencil computation each core would work on a distinct block of the lattice that needs to be held in cache. In addition, also neighboring sites (typically denoted as halos or ghost shells) need to be accessed, and thus be loaded into the cache. Without a shared cache this implies duplication

\footnotetext{
${ }^{3}$ The icc compiler does well in this respect for simple code parts like loops, but manual prefetches are beneficial for more complex parts like the application of the Dirac operator.
} 
at the L2 level: data for each lattice site may be present in the L2 caches of several cores. Similarly, the application code will by duplicated $N_{\text {core }} \sim 60$ times, since each core needs a copy of it. Finally, barriers are very expensive, up to 10,000 cycles for optimized implementations (see, e.g., an implementation by Intel as part of the QPhiX library [7]), while many standard implementations (such as in OpenMP and pthreads at the time of this writing) need well above 10,000 cycles.

As described above, a substantial fraction of each core's L2 is "wasted" for duplication of code and halos, thus reducing the fraction available for local data. Therefore we typically need a considerable amount of main-memory access. Stream benchmarks yield on the order of $170 \mathrm{~GB} / \mathrm{s}$ for the sum of read and write bandwidth (i.e., less than $50 \%$ of the nominal $352 \mathrm{~GB} / \mathrm{s}$ ). This bandwidth can be sustained for simple cases like streaming access thanks to the L2 hardware prefetcher. For more complex cases software prefetching may become necessary. The general memory access pattern is of importance as well. To obtain a bandwidth close to $170 \mathrm{~GB} / \mathrm{s}$, all cores must read and/or write simultaneously and continuously, i.e., if each core reads and/or writes in bursts (with breaks in between) the resulting overall bandwidth is much lower. Furthermore, if each core reads streams from more than about $5 \sim 10$ memory locations at a time, the overall bandwidth will not reach $170 \mathrm{~GB} / \mathrm{s}$, not even with manual L2 prefetching. This may be alleviated by modifying the data layout so that data needed at the same time are placed contiguously, e.g., by packing the gauge links for all four directions in one array instead of keeping a separate array for each direction.

For moving data over the network we typically rely on MPI, which in our case can use Intel's SCIF library (for intra-node communication) and/or the Infiniband HCA (for intra- and inter-node communication). SCIF shows good results in microbenchmarks: RDMA between two KNCs sustains a bidirectional bandwidth of about $6.5 \mathrm{~GB} / \mathrm{s}$ for payloads of $64 \mathrm{kB}$ and above, while PIO (programmed IO) sustains about $5 \mathrm{~GB} / \mathrm{s}$ for payloads of $16 \mathrm{kB}$ and above. These numbers are to be compared to the nominal value of $7.2 \mathrm{~GB} / \mathrm{s}$, which takes into account the overhead for the 256-Byte PCIe packages supported by the KNC [8]. The zero-size latencies are about $5.5 \mu$ s for RDMA and about $2 \mu$ s for PIO. However, in practice we experience issues: when using SCIF RDMA calls, the kernel seems to heavily utilize $5 \sim 10$ cores to do the data movement. These cores then become unusable for computation, since the application threads on these cores would slow down the whole machine. ${ }^{4}$ Intel MPI via the HCA, which utilizes the DMA engine of the HCA rather than that of the $\mathrm{KNC}$, does not show these issues. Another point is that the latency induced by the Intel MPI software stack is quite high. In microbenchmarks MVAPICH2-MIC seems to perform a bit better than Intel MPI (see also [9]), but at the time of this writing MVAPICH2-MIC always shows the SCIF issue explained above, even in cases where only the HCA should be used. In some performance-relevant parts we were successful in improving the performance by using Infiniband directly (i.e., without MPI) via the IB verbs library [10], at the cost of significantly increased complexity for the programmer.

\subsection{Application software}

We mainly use Chroma [11]. For the performance-relevant parts such as the DD-based solver

\footnotetext{
${ }^{4}$ One solution would be to leave these cores free, giving up $15 \%$ of the total performance, but even that is not enough: the kernel may occasionally schedule SCIF-related threads on one of the "compute" cores, again causing slowdown. In a big system this will of course happen very frequently. The situation could be improved by pinning these SCIF-related kernel threads to specific cores. This would be an interesting option for future work.
} 
described in Sec. 3 we wrote separate libraries that can be linked during Chroma compilation. The Wuppertal adaptive algebraic multigrid code $[12,13]$ has been ported to the KNC, including vectorization of all performance-relevant parts. In this code a Krylov solver is preconditioned with a V-cycle and a smoother. For the latter we use our DD preconditioner. Other parts of our code suite, such as HMC, hadron spectra, and hadron distribution amplitudes are also being adapted to the KNC. Furthermore, we made some improvements to QDP++ [11], such as memory management, optimization of existing threading, and some new threading. As for the actual implementation, the code is written in $\mathrm{C}++$, the performance-relevant parts are vectorized using icc intrinsics or auto-vectorization by the compiler, threading uses OpenMP, and the multi-node implementation is currently based on Intel MPI or IB verbs as described in Sec. 2.5.

\section{Domain decomposition on the Knights Corner processor}

Most of the content of this section is a summary of work done in collaboration with the Intel Parallel Computing Labs in Santa Clara (USA) and Bangalore (India) and with JLAB (USA), the details of which are described in [14]. This work was motivated by the first implementation of a conjugate-gradient-based solver on the KNC [15] (see also [16, 17, 18] for other implementations). The strong-scaling behavior of that solver on a production system (TACC Stampede) turned out to be rather limited, the performance bottleneck being the bandwidths for memory access and internode communication. The obvious way to improve upon the strong-scaling behavior is to switch to another algorithm, based on domain decomposition, that moves less data between KNC and memory, and between different KNCs.

In fact, this is a good example of how the implementation of high-performance application code on a new hardware architecture can proceed. First, one identifies the most suitable algorithm for the given hardware architecture. Second, one identifies the most suitable data layout for the given algorithm and hardware architecture. Only then should one proceed to optimize the code (including vectorization, threading, parallelization, etc.).

\subsection{Algorithm and implementation overview}

Let us briefly describe some details of our algorithm. For the Dirac operator we use WilsonClover. Our outer solver is flexible GMRES with deflated restarts [19]. As a preconditioner we use the multiplicative Schwarz method (a.k.a. Schwarz alternating procedure), which was introduced in [20] and adapted to Lattice QCD in [4]. The main idea of the Schwarz method is to subdivide the lattice into domains. After a reordering of indices, the matrix to be inverted then consists of a block-diagonal part (corresponding to the interactions within the domains) and a remainder (corresponding to the interactions between domains). Based on this splitting, a block-Jacobi iteration can be performed to approximate the inverse. If the domains are small enough to fit in cache, the ensuing inversion on the domains (i.e., the block-diagonal parts) can be done without off-chip data movement. ${ }^{5}$ Computation of the remainder does require communication but occurs less frequently. As a result, the overall algorithm needs less communication bandwidth, is more latency tolerant, and gives more cache reuse than the conjugate-gradient algorithm.

\footnotetext{
${ }^{5}$ For the inversion on the domains we use the minimal-residual (MR) algorithm with even-odd preconditioning.
} 


\subsection{Data layout and vectorization}

For optimal performance it is necessary (i) to fully utilize the SIMD units, (ii) to choose the data layout such that the cache lines loaded into the KNC do not contain unnecessary data, and (iii) to avoid instruction overheads due to SIMD vector permutations. Typically one cannot satisfy all criteria simultaneously and tries to find a compromise that maximizes the overall performance. In our case, the best solution is a structure-of-array (SOA) format in which all 24 floating-point components of a spinor are stored in 24 separate registers and cache lines. This leads to "site fusing", i.e., one 512-bit register contains data from 16 sites (in single precision). Since we have one small domain per core (see Sec. 3.3) we have to site-fuse in more than one dimension. For example, for an $8 \times 4^{3}$ domain we site-fuse the $x$ - and $y$-dimensions, with data from $4 \times 4$ sites in each register. ${ }^{6}$

In this scheme, the computation of hopping terms within domains can be done straightforwardly in the $z$ - and $t$-directions for complete registers, while in the site-fused directions some permute and mask instructions are needed that lead to an underutilization of the SIMD units of $12.5 \%$ in the $x$ - and $25 \%$ in the $y$-direction. For the computation of hopping terms between domains, again the $z$ - and $t$-directions are straightforward, while in the site-fused directions the cache lines that need to be loaded to access the neighbor's boundary data contain many data that are not needed. We avoid this overhead by additionally storing the boundary data in an array-of-structure (AOS) format [14].

\subsection{Cache management and prefetching}

Since the L2 is not shared we assign each domain to a single core. The cache size of $512 \mathrm{kB}$ per core restricts the domain size to $8 \times 4^{3}$ (in single precision). We actually implemented domains of size $4^{4}$ and $8 \times 4^{3}$. Since the KNC can do up- and down-conversion, we store some domain data, i.e., the gauge links and clover matrices, in half precision. This reduces the working set (from $456 \mathrm{kB}$ to $312 \mathrm{kB}$ per $8 \times 4^{3}$ domain) and the memory-bandwidth requirements. To ensure stability, we keep the spinors in single precision. We observed that this optimization had no noticeable impact on the iteration count of the outer solver.

As discussed in Sec. 2.5, the KNC has no L1 hardware prefetcher, and an L2 hardware prefetcher only for streaming access. Because of the irregular code structure, compiler-generated software prefetches are often not good enough. It is therefore essential to manually insert L1 and L2 prefetches using intrinsics. The fine-tuning of these prefetches turned out to be rather timeconsuming.

\subsection{Intra-core threading}

As mentioned in Sec. 2.5, at least two threads per core are necessary to fully utilize the floatingpoint unit. In our implementation we assign threads to alternating time slices within a domain. There are pros and cons for using two or four threads per core. Two threads suffer from more stalls since L1 or L2 misses cannot be hidden by other threads. Four threads suffer from more L1 conflict misses because our working set exhausts the L1 size. We measured the performance for both cases and found no significant differences.

\footnotetext{
${ }^{6}$ Our notation is always $L_{x} \times L_{y} \times L_{z} \times L_{t}$, i.e., $8 \times 4^{3}$ means $L_{x}=8$ and $L_{y}=L_{z}=L_{t}=4$.
} 


\subsection{Inter-core parallelization}

When parallelizing over the cores of the KNC, synchronization between cores is only necessary after an MR block solve, which implies that the cost of a barrier (which can be quite large on the KNC, see Sec. 2.5) has little impact on the overall performance.

A simple but very performance-relevant issue is load balancing. Standard lattice sizes are typically powers of 2 and thus do not map naturally to 60 or 61 cores. As a result, some of the cores are unused at least part of the time ("load imbalance"). A simple solution would be to include factors of 3 and 5 in the lattice size, but this is only an option for new simulations where we can select the lattice size. Another option is to increase the local volume (per KNC) so that the number $N_{\text {domain }}$ of domains assigned to a single $\mathrm{KNC}^{7}$ is much larger than the number $N_{\text {core }}$ of cores per KNC. The average load is then given by the function $x /$ ceil $(x)$ with $x=N_{\text {domain }} / 2 N_{\text {core }}$ [14], which has a saw-tooth-like behavior with the minima approaching 1 from below as $N_{\text {domain }}$ increases. However, increasing the local volume is not an option in the strong-scaling region for which we are trying to optimize. The third option is a non-uniform distribution of the lattice over multiple KNCs (assuming a multi-node implementation as described below). Let us give a simple example to illustrate the idea, using a lattice size of $64^{3} \times 128$ and a domain size of $8 \times 4^{3}$. If the lattice is uniformly distributed over, say, $1024 \mathrm{KNCs}$ in a $4 \times 4 \times 8 \times 8$ layout, we have $N_{\text {domain }}=64$ and thus a load of 53\%. In that case each KNC contains 16 sites in the $t$-direction. Alternatively, we could split the $t$-direction non-uniformly as $128=4 \cdot 28+16$ so that we need only 5 (instead of 8) KNCs for the $t$-direction. This reduces the number of KNCs from 1024 to 640 and increases the average load to $(4 \cdot 56+32) /(5 \cdot 60)=85 \%$. The time to solution will not decrease (and may in fact increase slightly because the boundaries to be communicated will be larger), but the cost to solution will decrease by almost $40 \%$.

\subsection{Multi-node implementation}

For the parallelization over many KNCs using MPI we have several options. For example, each thread could issue its own MPI calls. However, this option has two disadvantages. First, the overhead of many simultaneous MPI calls is high in typical MPI implementations. Second, the packets generated by each thread are too small to efficiently utilize the network. Therefore, a better option is to combine the surface data of all domains and to communicate them using only a single thread. This option also has a disadvantage, i.e., the need for explicit on-chip synchronization, but in practice it performs better than the other option.

As in any parallelization effort, it is essential to overlap computation and communication in order to hide the communication latencies. The standard method is to divide the local volume into interior and surface and to work on the interior while waiting for the surface communication. For our domain-decomposition approach this does not work for typical parameters since most domains would be on the surface, i.e., we have only a small interior, or none at all. Instead, we devised a new method, see Fig. 8. In the left part we first show a splitting in a single direction, here $t$. Computations are done on each time-slice in the order indicated by the numbers. Boundary data

\footnotetext{
${ }^{7}$ Note that the number of domains that can be processed independently is given by $n_{\text {domain }}=N_{\text {domain }} / 2$. The factor of 2 is due to the checkerboarding in the multiplicative Schwarz method.

${ }^{8}$ Here, we use $N_{\text {core }}=60$ since Intel configures the Linux kernel to run on the last core.
} 

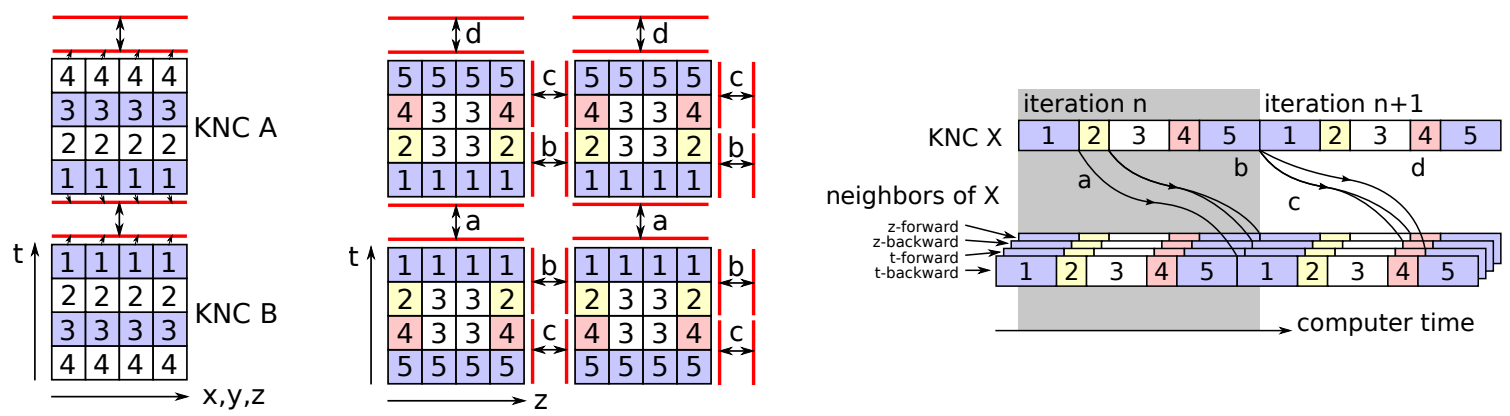

Figure 8: Illustration of our method to hide communication latencies. Each little box represents a domain. Left: Splitting only in the $t$-direction. Middle: Splitting in $t$ - and $z$-direction. Right: Linear representation of the scheme in the middle. See text for details.

can be sent after (1) has finished. These data are only needed for (1) in the next iteration. Thus, the communication of (1) overlaps with the computation of (2)-(4). However, this only works for $t$, but not for the other directions because their boundaries can only be sent after (4) has finished. A better scheme is shown in the middle and right parts. Here, we split in the $t$ - and $z$-directions (in practice we split in all directions, and the same principle applies). Again, the order of the computations is indicated by the numbers, while the order of the communications is indicated by letters. As before, (a) occurs after (1), but in the $z$-direction we now send boundary data when the computation on half of the boundary has finished, i.e., (b) occurs after (2). The other half of the boundary is sent at the end, i.e., (c) occurs after (5). The first half is needed at the start of the next iteration, which means that (b) overlaps with (3)-(5). The second half is needed before (4) of the next iteration so that (c) then overlaps with (1)-(3).

\subsection{Results}

In this subsection we discuss the performance of our code for three cases: single core, single $\mathrm{KNC}$, and many KNCs. Since we are interested in realistic lattice sizes, for which the lattice is distributed over many KNCs, the relevant parts of the code are MR iteration and DD preconditioner for the single-core and single-KNC cases, and full solver for the many-KNC case.

We first present some theoretical single-core performance estimates for the Wilson-Clover operator, all in single precision. We assume that our domains fit in cache so that we are not memorybandwidth bound. Of the 1848 flops/site in Wilson-Clover, 64\% are fused multiply-adds, which gives an upper performance limit of $82 \%$. Instruction overheads due to masking, shuffles and permutes as well as imperfect instruction pairing reduce the theoretical limit to $56 \%$ or $22 \mathrm{Gflop} / \mathrm{s} / \mathrm{core}$, see [14] for details. Our measured performance for the MR iteration is about $13.3 \mathrm{Gflop} / \mathrm{s} / \mathrm{core}$. According to VTune (Intel's performance-analysis tool) this performance loss is mainly due to stalls because of outstanding L1 prefetches. Since the optimal number of MR iterations in terms of time-to-solution is only $4 \sim 5$, the Schwarz method is not completely dominated by MR, i.e., other parts contribute noticeably, and the overall performance of the Schwarz method drops to 9.5 Gflops/s/core. Table 1 shows the impact of our optimization efforts on the performance numbers.

Let us now discuss the many-core performance of the DD preconditioner when parallelizing over 60 cores of the KNC, see Fig. 9. We clearly observe the load-balancing issue discussed in Sec. 3.5. As long as the local volume and thus the number of domains is large enough, the 


\begin{tabular}{l|cc|cc} 
& \multicolumn{2}{|c|}{ MR iteration } & \multicolumn{2}{c}{ DD method } \\
& single & half & single & half \\
\hline no software prefetching & 6.1 & 8.9 & 4.6 & 6.6 \\
L1 prefetches & 10.4 & 13.3 & 6.5 & 8.7 \\
L1+L2 prefetches & 10.2 & 13.3 & 7.1 & 9.5 \\
\hline
\end{tabular}

Table 1: Single-core performance in Gflop/s (single precision) for the 7120X. See text for details.

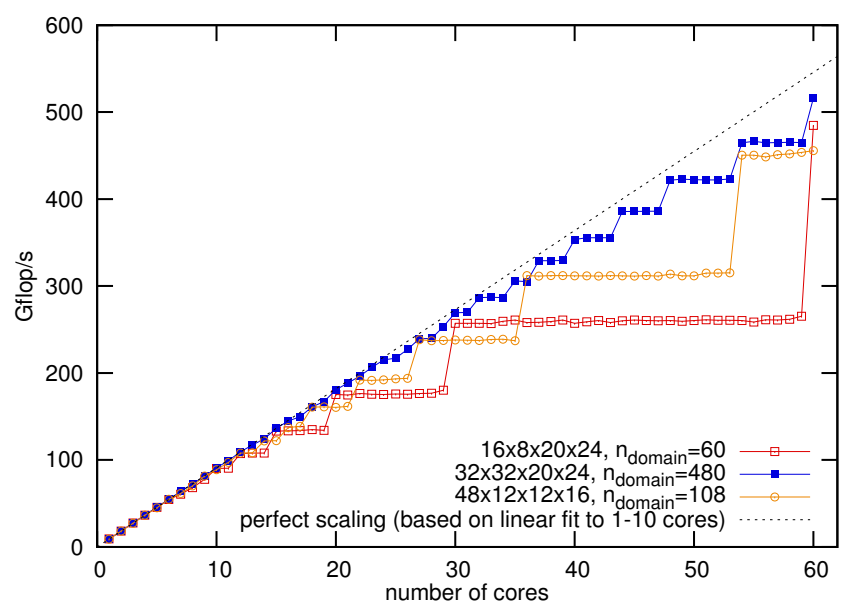

Figure 9: On-chip strong-scaling of the DD preconditioner (with $5 \mathrm{MR}$ iterations and 16 Schwarz iterations). The first two volumes are chosen such that $n_{\text {domain }}$ is divisible by 60 , i.e., on 60 cores we have $100 \%$ load. The other volume corresponds to distributing a $48^{3} \times 64$ lattice over $64 \mathrm{KNCs}$, with a load of $90 \%$ on 60 cores.

effect is small, but once $N_{\text {domain }}$ gets close to $N_{\text {core }}$ it becomes serious. Apart from this effect the scaling is almost linear, because (i) during the MR inversion the cores run independently from their respective caches, i.e., memory bandwidth does not become a bottleneck, and (ii) the other (i.e., non-MR) parts of the DD method, which do require synchronization, are sub-dominant.

Since the full QPACE 2 machine is not yet available, we performed multi-node benchmarks of the complete solver on up to 1024 nodes of the TACC Stampede cluster, which uses the 7110P version of the KNC. Due to the limited space we do not give a detailed discussion here but refer to [14] for details. As an example, we briefly discuss the results for a $64^{3} \times 128$ lattice. In the strong-scaling case relevant for HMC, the non-DD solver of [15] levels off at about $128 \mathrm{KNCs}$, while our DD solver still scales roughly linearly up to the maximum partition size of $1024 \mathrm{KNCs}$, with a time-to-solution gain of about a factor of 5. In the weak-scaling (or minimum-cost) case relevant for data analysis, our DD solver outperforms the non-DD solver by about a factor of $2 \sim 3$.

\section{Summary and outlook}

We have presented the architecture of QPACE 2, a massively parallel and very densely packed system of KNC processors coupled by a two-layer network based on PCIe and FDR Infiniband. Two complete bricks have been running synthetic benchmarks as well as lattice QCD simulations continuously for over a month without errors. The full machine is currently (February 2015) being 
assembled and tested in Regensburg and will enter production mode soon. High-performance solver code based on domain decomposition as well as application code is already available, see Sec. 2.6. The plan is to make most of our codes publicly available in the near future.

The design of QPACE 2 allows for an upgrade to the next version of the Xeon Phi processor, code-named Knights Landing (KNL). Compared to the KNC, this processor will have three times the floating-point performance at the same or even less power, better cores (with out-of-order execution and hardware prefetcher), high-bandwidth memory integrated on the package, a PCIe Gen3 interface, and in some versions an integrated network controller (Omni-Path). A follow-up project, QPACE 3, will use the KNL processor and have a target peak performance of about 4 Pflop/s in double precision. The plan is to install this system at the Jülich Supercomputing Center in 2016.

We thank the German Research Foundation (DFG) for the funding that made this project possible, the machine shop and the electronics lab of the Regensburg University Physics Department for their contributions to the design, and T-Platforms for their support at an early stage of the project.

\section{References}

[1] K. G. Wilson, Phys.Rev. D10 (1974) 2445.

[2] T. Wettig, PoS LAT2005 (2006) 019 [hep-lat/ 0509103$].$

[3] H. Baier et al., PoS LAT2009 (2009) 001 [arXiv: 0911.2174 ].

[4] M. Luscher, Comput.Phys.Commun. 156 (2004) 209 [hep-lat/ 0310048 ].

[5] Y. Iwasaki, Nucl.Phys.Proc.Suppl. 34 (1994) 78 [hep-lat/ 9401030 ].

[6] J. Beddoes and M. Bibby, Principles of Metal Manufacturing Processes. Elsevier Science (1999).

[7] https://github.com/JeffersonLab/qphix.git.

[8] http://www.plxtech.com/pdf/technical/expresslane/Choosing_PCIe_Packet_Payload_Size.pdf.

[9] S. Potluri et al., SC'13 Proceedings of the International Conference for High Performance Computing, Networking, Storage and Analysis, ACM, New York (2013) 54.

[10] https://www.openfabrics.org/downloads/libibverbs.

[11] SciDAC, LHPC, and UKQCD Collaborations (R. G. Edwards and B. Joó), Nucl.Phys.Proc.Suppl. 140 (2005) 832 [hep-lat/0409003].

[12] A. Frommer, K. Kahl, S. Krieg, B. Leder, and M. Rottmann, SIAM J.Sci.Comput. 36 (2014) A1581 [arXiv:1303.1377].

[13] A. Frommer, K. Kahl, S. Krieg, B. Leder, and M. Rottmann, arXiv:1307.6101.

[14] S. Heybrock et al., SC '14 Proceedings of the International Conference for High Performance Computing, Networking, Storage and Analysis, IEEE Press (2014) 69 [arXiv: 1412.2629].

[15] B. Joó et al., Proceedings of ISC '13, Lecture Notes in Computer Science 7905 (2013) 40.

[16] R. Li and S. Gottlieb, arXiv:1411.2087.

[17] Y.-C. Jang et al., arXiv:1411.2223.

[18] O. Kaczmarek, C. Schmidt, P. Steinbrecher, and M. Wagner, arXiv:1411.4439.

[19] A. Frommer, A. Nobile, and P. Zingler, arXiv:1204.5463.

[20] H. A. Schwarz, Vierteljahrsschrift der Naturforschenden Gesellschaft in Zürich 15 (1870) 272. 